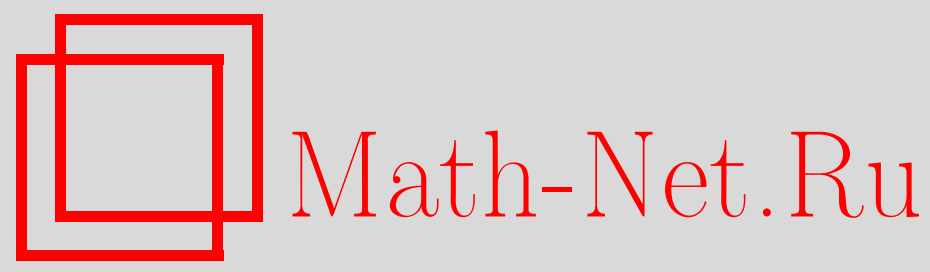

А. М. Блохин, Р. Е. Семенко, Вихревые стационарные структуры Кармана в магнитогидродинамических течениях вращающейся несжимаемой полимерной жидкости, $\mathrm{Ma}$ тем. моделирование, 2020, том 32, номер 7, 3-23

DOI: https://doi.org/10.20948/mm-2020-07-01

Использование Общероссийского математического портала Math-Net.Ru подразумевает, что вы прочитали и согласны с пользовательским соглашением http://www . mathnet.ru/rus/agreement

Параметры загрузки:

IP : 3.82 .47 .9

26 апреля 2023 г., $14: 27: 43$

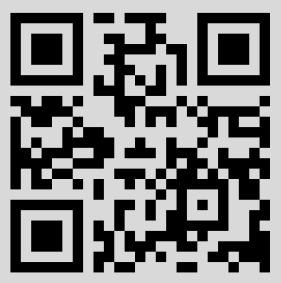




\title{
ВИХРЕВЫЕ СТАЦИОНАРНЫЕ СТРУКТУРЫ КАРМАНА В МАГНИТОГИДРОДИНАМИЧЕСКИХ ТЕЧЕНИЯХ ВРАЩАЮЩЕЙСЯ НЕСЖИМАЕМОЙ ПОЛИМЕРНОЙ ЖИДКОСТИ
}

(C) 2020 2. A.М. Блохин ${ }^{1,2}$, Р.Е. Семенко ${ }^{1,2}$

${ }^{1}$ Новосибирский государственный университет

${ }^{2}$ Институт математики им. С.Л. Соболева, Новосибирск

blokhin@math.nsc.ru; r.semenko@g.nsu.ru

Работа выполнена при поддержке программы фундаментальных научных исследований СО РАН I.1.5, проект 0314-2016-0013.

DOI: $10.20948 / \mathrm{mm}-2020-07-01$

Рассматриваются стационарные решения для задачи о магнитогидродинамическом движении несжимаемой полимерной жидкости над бесконечным вращающимся диском. Мы используем представление решения, аналогичное автомодельному решению Кармана для вязкой жидкости. Приводятся примеры численных стационарных решений для различных значений параметров магнитного поля.

Ключевые слова: реологическая модель, вращающееся движение, магнитная гидродинамика, стационарные решения.

\section{STATIONARY "von KARMAN" VORTEX STRUCTURES \\ IN THE MAGNETOHYDRODYNAMICAL FLOWS \\ OF ROTATING INCOMPRESSIBLE POLYMERIC LIQUID}

\author{
A.M. Blokhin ${ }^{1,2}$, R.E. Semenko ${ }^{1,2}$ \\ ${ }^{1}$ Novosibirsk State University, Novosibirsk \\ ${ }^{2}$ Sobolev Institute of Mathematics SB RAS, Novosibirsk
}

We study the steady-state solutions for the problem of magnetohydrodyamical flow of incompressible polymeric liquid above the infinite flat disc. In the paper we are looking for the solutions similar to the von Karman self-similar solutions for the viscous liquid. We intoduce the examples of the numerical steady-state solutions for the various values of problem parameters.

Keywords: rheological model, rotating motion, magnetohydrodynamics, steady-state solutions.

\section{Введение}

Построение и исследование моделей растворов и расплавов полимерных материалов является важной современной задачей в связи с очевидно 
широким использованием полимеров в самых разных областях промышленности. Однако текучие полимерные среды с трудом поддаются точному моделированию из-за своей комплексной макромолекулярной структуры. Экспериментальные данные по изучению жидких полимеров демонстрируют сложное и разнообразное поведение таких жидкостей и мало предсказуемые нелинейные зависимости параметров течения от концентрации полимера в растворе, температуры, молекулярной массы и прочих макро- и микрохарактеристик материала. На сегодняшний день существует большое количество разработанных моделей, но из-за такого большого разнообразия наблюдаемых физических свойств ни одна из существующих моделей не охватывает весь спектр характерных свойств динамики жидких полимеров, и пока нет оснований предполагать, что таковые появятся в будущем. В связи с этим, существующие на данный момент модели требуют тщательного теоретического анализа с целью понимания границ их применимости и свойств имеющихся у них решений.

Магнитная гидродинамика вязкоупругих неньютоновских жидкостей, в том числе растворов и расплавов полимеров, на сегодняшний день активно исследуется с точки зрения различных приложений (материалы, магнитогидродинамические генераторы, электростатические фильтры и др.). При этом вышеупомянутое разнообразие математических моделей полимерных сред диктует необходимость разработки и исследования магнитогидродинамических приближений отдельно для каждой такой модели. Существует широкий круг работ по изучению МГД уравнений для обобщенных ньютоновских жидкостей (напр., [1]), для среды Олдройда [2-4]. Так, в частности, в [4,5] изучались вращательные МГД течения жидкости в рамках широко распространенной феноменологической модели Олдройд-Б. В статье [6] нами предлагался вариант магнитогидродинамической модификации обобщенной модели Виноградова-Покровского [7] с учетом потока тепла. Однако вращательные МГД течения в рамках этой модели еще не изучались.

Целью данной работы является изучение магнитогидродинамического аналога классического вращательного движения жидкости, расположенной над бесконечным вращающимся диском. Постановка подобной задачи для вязкой ньютоновской жидкости и нахождение стационарных решений задачи восходят к знаменитой работе Теодора фон Кармана [8]. В дальнейшем исследование этой задачи активно развивалось усилиями множества авторов ([9-12] и др.). Нами предлагается рассмотреть течение аналогичной геометрии для вязкоупругой полимерной жидкости в присутствии магнитного поля. Помимо этого, существенным отличием постановки нашей задачи от 
классической постановки Кармана является конечная толщина слоя жидкости, что роднит задачу с рассмотренной в $[11,12]$. Причем в нашем случае эта толщина является величиной, подлежащей определению. Приближенные стационарные решения задачи мы ищем в форме, сходной с предложенным Карманом автомодельным решением [8]. Основной задачей работы мы видим вычисление стационарных решений для различных параметров задачи и исследование поведения этих решений в зависимости от характеристик магнитного поля.

\section{1. Предварительные сведения}

Рассмотрим магнитогидродинамическое течение вязкоупругой несжимаемой полимерной жидкости над вращающимся диском бесконечного радиуса. Общая схема рассматриваемого нами течения аналогична той, что изучалась, например, в [11,12]: бесконечный по горизонтали слой несжимаемой жидкости плотности $\rho=$ const находится над горизонтальной пластиной, вращающейся с угловой скоростью $\Omega \Lambda(t)$. Здесь $\Omega=$ const - размерный параметр, $\Lambda(t)$ - безразмерная функция. Слой ограничен сверху свободной поверхностью. Допустим также, что область течения помещена в магнитное поле напряженности $\mathbf{H}=(\tilde{L}, \tilde{M}, 1+\tilde{N})$, где компоненты вектора напряженности записаны в цилиндрических координатах $(r, \phi, z)$, см. рис.1. Предполагая наличие слабого электромагнитного поля, вектор магнитной индукции В мы будем брать в виде [13]:

$$
\mathbf{B}=\mu \mu_{0} \mathbf{H}=(1+\chi) \mu_{0} \mathbf{H},
$$

где $\mu_{0}$ - магнитная проницаемость в вакууме, $\mu$ - магнитная проницаемость среды, $\chi$ - магнитная восприимчивость, причем $\chi=\chi_{0} T_{0} / T, \chi_{0}-$ магнитная восприимчивость при комнатной температуре $T_{0}=(300 \mathrm{~K}), T-$ температура среды [14]. Также будем полагать, что для полимерной жидкости (в области $S$, см. рис.1) $\mu \equiv 1\left(\chi_{0}=0\right)$. Заметим, что из-за магнитного поля, при вращении металлического диска возникает электродвижущая сила индукции [15]. Но поскольку электрическая цепь разомкнута, электрического тока в металлическом диске не будет.

В отличие от работ [8,10-12], нами рассматривается течение вязкоупругой полимерной жидкости, что требует привлечения реологической модели. Для моделирования динамики таких жидкостей необходимо ввести определяющее соотношение, связывающее значения компонент симметрического тензора напряжений и тензора градиента поля скоростей. В отличие от вязкой ньютоновской жидкости, эта зависимость не является линейной. Более 
того, ключевой особенностью вязкоупругих жидкостей является наличие памяти деформаций. Другими словами, напряжения среды в некоторый момент времени зависят не только от состояния жидкости в этот же момент, но и от истории течения. Масштаб этой временной зависимости характеризуется таким параметром, как время релаксации. Таким образом, определяющее соотношение не может быть выражено в явном виде и должно представлять из себя систему интегральных или дифференциальных уравнений по времени.

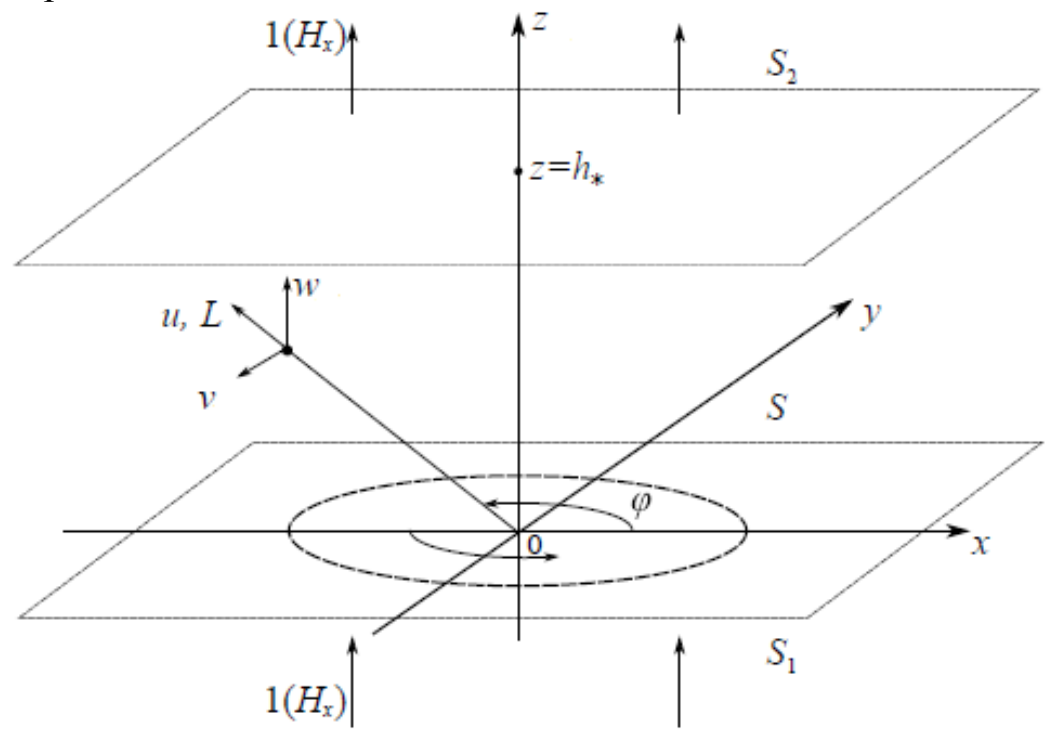

Рис.1. Зона вращающегося движения.

Дадим краткое описание реологической модели Виноградова-Покровского [7], которую мы используем в данной работе. Эта модель относится к классу мезоскопических, т.е. для получения соотношений она описывает движение макромолекулы жидкости в потоке. Сама макромолекула моделируется системой броуновских частиц - бусинок, соединенных упругими сегментами - пружинками. Реакция окружающей среды на молекулу описывается анизотропной вязкоупругой средой. В силу этого, в правых частях уравнений законов сохранения появляются тензорные коэффициенты, характеризующие вязкие напряжения, упругость и релаксацию. В рамках модели Виноградова-Покровского делается предположение, что эти коэффициенты можно выразить через так называемый тензор анизотропии $\Pi=\left\{a_{i j}\right\}, i, j,=r, \phi, z$ - безразмерный тензор вторых моментов нормальных координат сегментов макромолекулы $[16,17]$. Если дополнительно допустить, что тензорные коэффициенты в законах сохранения выражаются через тензор анизотропии 
линейно, что имеет смысл для случаев малой анизотропии, то вклад анизотропии в динамику жидкости будет характеризоваться двумя дополнительными скалярными величинами - постоянными феноменологическими параметрами $k$ и $\beta$. При этом параметр $k$ учитывает вклад, связанный с размерами макромолекул, а $\beta$ - вклад, связанный с их ориентацией.

Таким образом, неизвестными величинами модели с учетом магнитного поля являются вектор скорости $\mathbf{u}=(u, v, w)$ в цилиндрической системе координат, давление $P$, шесть компонент тензора анизотропии и три компоненты вектора магнитной индукции. Уравнения мы приведем в безразмерном виде, при этом в качестве размерных величин мы будем использовать характерную длину $l_{H}$, скорость $u_{H}=\Omega l_{H}$, время $l_{H} / u_{H}$, давление $\rho u_{H}^{2}$ и характерную величину напряженности магнитного поля $H_{x}$. Процесс обезразмеривания аналогичен описанному в [18-20].

Приступим теперь к обсуждению уравнений модели. Уравнение неразрывности в цилиндрических координатах имеет стандартный вид

$$
\operatorname{div} \mathbf{u}=\frac{\partial u}{\partial r}+\frac{1}{r} \frac{\partial v}{\partial \phi}+\frac{\partial w}{\partial z}+\frac{u}{r}=0
$$

Как было отмечено ранее, уравнения закона сохранения импульса для вязкоупругой жидкости имеют правые части, зависящие от анизотропии среды. С учетом вязких напряжений и релаксации, а также с учетом объемных сил, действующих со стороны магнитного поля, эти уравнения принимают следующий вид (см. [7,13,21-23]):

$$
\begin{aligned}
& \frac{d u}{d t}-\frac{v^{2}}{r}+\frac{\partial P}{\partial r}=\frac{1}{\operatorname{Re}}\left(\frac{\partial a_{r r}}{\partial r}+\frac{1}{r} \frac{\partial a_{r \phi}}{\partial \phi}+\frac{\partial a_{r z}}{\partial z}+\frac{a_{r r}-a_{\phi \phi}}{r}\right)+ \\
& +\sigma_{m}\left(\frac{\tilde{M}}{r} \frac{\partial \tilde{L}}{\partial \phi}+(1+\tilde{N})\left(\frac{\partial \tilde{L}}{\partial z}-\frac{\partial \tilde{N}}{\partial r}\right)-\tilde{M} \frac{\partial \tilde{M}}{\partial r}-\frac{\tilde{M}^{2}}{r}\right) \\
& \frac{d v}{d t}+\frac{u v}{r}+\frac{1}{r} \frac{\partial P}{\partial \phi}=\frac{1}{\operatorname{Re}}\left(\frac{\partial a_{r \phi}}{\partial r}+\frac{1}{r} \frac{\partial a_{\phi \phi}}{\partial \phi}+\frac{\partial a_{\phi z}}{\partial z}+\frac{2}{r} a_{r \phi}\right)+ \\
& +\sigma_{m}\left(\tilde{L} \frac{\partial \tilde{M}}{\partial r}-(1+\tilde{N})\left(\frac{\partial \tilde{M}}{\partial z}-\frac{1}{r} \frac{\partial \tilde{N}}{\partial \phi}\right)+\frac{\tilde{L}}{r}\left(\tilde{M}-\frac{\partial \tilde{L}}{\partial \phi}\right)\right) \\
& \frac{d w}{d t}+\frac{\partial P}{\partial z}=\frac{1}{\operatorname{Re}}\left(\frac{\partial a_{r z}}{\partial r}+\frac{1}{r} \frac{\partial a_{\phi z}}{\partial \phi}+\frac{\partial a_{z z}}{\partial z}+\frac{a_{r z}}{r}\right)+ \\
& +\sigma_{m}\left(\tilde{L}\left(\frac{\partial \tilde{N}}{\partial r}-\frac{\partial \tilde{L}}{\partial z}\right)+\frac{\tilde{M}}{r} \frac{\partial \tilde{N}}{\partial \phi}-\tilde{M} \frac{\partial \tilde{M}}{\partial z}\right) .
\end{aligned}
$$


Здесь $t$ - время, $\sigma_{m}=\left(\mu \mu_{0} H_{x}^{2}\right) /\left(\rho u_{H}^{2}\right)-$ коэффициент магнитного давления. Оператор полной производной по времени выглядит так:

$$
\frac{d}{d t}=\frac{\partial}{\partial t}+u \frac{\partial}{\partial r}+\frac{v}{r} \frac{\partial}{\partial \phi}+w \frac{\partial}{\partial z}
$$

Уравнения Максвелла данной модели возьмем в виде [13,21]:

$$
\begin{gathered}
\operatorname{div} \mathbf{H}=\frac{1}{r} \frac{\partial(r \tilde{L})}{\partial r}+\frac{1}{r} \frac{\partial \tilde{M}}{\partial \phi}+\frac{\partial \tilde{H}}{\partial z}=0 \\
\frac{d \tilde{L}}{d t}-\left(\tilde{L} \frac{\partial u}{\partial r}+\frac{\tilde{M}}{r} \frac{\partial u}{\partial \phi}+(1+\tilde{N}) \frac{\partial u}{\partial z}\right)-b_{m}\left(\Delta_{r, \phi, z} \tilde{L}-\frac{2}{r^{2}} \frac{\partial \tilde{M}}{\partial \phi}-\frac{\tilde{L}}{r^{2}}\right)=0 \\
\frac{d \tilde{M}}{d t}+\frac{v \tilde{L}}{r}-\left(\tilde{L} \frac{\partial v}{\partial r}+\frac{\tilde{M}}{r} \frac{\partial v}{\partial \phi}+(1+\tilde{N}) \frac{\partial v}{\partial z}+\frac{\tilde{M} u}{r}\right)-b_{m}\left(\Delta_{r, \phi, z} \tilde{M}+\frac{2}{r^{2}} \frac{\partial \tilde{L}}{\partial \phi}-\frac{\tilde{M}}{r^{2}}\right)=0 \\
\frac{d \tilde{N}}{d t}-\left(\tilde{L} \frac{\partial w}{\partial r}+\frac{\tilde{M}}{r} \frac{\partial w}{\partial \phi}+(1+\tilde{N}) \frac{\partial w}{\partial z}\right)-b_{m} \Delta_{r, \phi, z} \tilde{N}=0
\end{gathered}
$$

Здесь $b_{m}=1 / \operatorname{Re}_{m}, \operatorname{Re}_{m}=\sigma \mu \mu_{0} u_{H} l_{H}$ - магнитное число Рейнольдса, $\sigma$ - электропроводность среды. Оператор Лапласа $\Delta_{r, \phi, z}$ равен

$$
\Delta_{r, \phi, z}=\frac{\partial^{2}}{\partial r^{2}}+\frac{1}{r^{2}} \frac{\partial^{2}}{\partial \phi^{2}}+\frac{\partial^{2}}{\partial z^{2}}+\frac{1}{r} \frac{\partial}{\partial r} .
$$

Приведенные уравнения замыкаются определяющим соотношением, которое для данной модели представляет из себя шесть дифференциальных уравнений [7]:

$$
\begin{aligned}
& \frac{d a_{r r}}{d t}-2\left(A_{r} \frac{\partial u}{\partial r}+\frac{a_{r \phi}}{r} \frac{\partial u}{\partial \phi}+a_{r z} \frac{\partial u}{\partial z}\right)+R_{r r}=0 \\
& \frac{d a_{\phi \phi}}{d t}+2\left(\frac{v}{r}-\frac{\partial v}{\partial r}\right) a_{r \phi}-2\left(\frac{1}{r}\left(u+\frac{\partial v}{\partial \phi}\right) A_{\phi}+a_{\phi z} \frac{\partial v}{\partial z}\right)+R_{\phi \phi}=0 \\
& \frac{d a_{z z}}{d t}-2\left(a_{r z} \frac{\partial w}{\partial r}+\frac{a_{\phi z}}{r} \frac{\partial w}{\partial \phi}+A_{z} \frac{\partial u}{\partial z}\right)+R_{z z}=0, \\
& \frac{d a_{r \phi}}{d t}+\left(\frac{v}{r}-\frac{\partial v}{\partial r}\right) A_{r}+\left(a_{r \phi} \frac{\partial w}{\partial z}-a_{r z} \frac{\partial v}{\partial z}-\frac{1}{r} A_{\phi} \frac{\partial u}{\partial \phi}-a_{\phi z} \frac{\partial u}{\partial z}\right)+R_{r \phi}=0,
\end{aligned}
$$




$$
\begin{aligned}
& \frac{d a_{r z}}{d t}-a_{r z}\left(\frac{\partial u}{\partial r}+\frac{\partial w}{\partial z}\right)-\left(A_{r} \frac{\partial w}{\partial r}+\frac{a_{r \phi}}{r} \frac{\partial w}{\partial \phi}+\frac{a_{\phi z}}{r} \frac{\partial u}{\partial \phi}+A_{z} \frac{\partial u}{\partial z}\right)+R_{r z}=0 \\
& \frac{d a_{\phi z}}{d t}+\left(\frac{v}{r}-\frac{\partial v}{\partial r}\right) a_{r z}-\left(a_{\phi z} \frac{\partial u}{\partial r}+A_{z} \frac{\partial v}{\partial z}+a_{r \phi} \frac{\partial w}{\partial r}+\frac{A_{\phi}}{r} \frac{\partial w}{\partial \phi}\right)+R_{\phi z}=0
\end{aligned}
$$

Здесь

$$
\begin{aligned}
& R_{r r}=K_{I} a_{r r}+\beta\left\|\mathbf{a}_{r}\right\|^{2}, \quad \mathbf{a}_{r}=\left(a_{r r}, a_{r \phi}, a_{r z}\right), \quad R_{\phi \phi}=K_{I} a_{\phi \phi}+\beta\left\|\mathbf{a}_{\phi}\right\|^{2}, \mathbf{a}_{\phi}=\left(a_{r \phi}, a_{\phi \phi}, a_{\phi z}\right), \\
& R_{z z}=K_{I} a_{z z}+\beta\left\|\mathbf{a}_{z}\right\|^{2}, \quad \mathbf{a}_{z}=\left(a_{r z}, a_{\phi z}, a_{z z}\right), \quad R_{r \phi}=K_{I} a_{r \phi}+\beta\left(\mathbf{a}_{r}, \mathbf{a}_{\phi}\right), \quad R_{r z}=K_{I} a_{r z}+ \\
& +\beta\left(\mathbf{a}_{r}, \mathbf{a}_{z}\right), \quad R_{\phi z}=K_{I} a_{\phi z}+\beta\left(\mathbf{a}_{\phi}, \mathbf{a}_{z}\right), \quad\left\|\mathbf{a}_{r}\right\|^{2}=\left(\mathbf{a}_{r}, \mathbf{a}_{r}\right) \quad \text { и т.д., } \quad A_{r}=a_{r r}+\mathrm{W}^{-1}, \\
& A_{\phi}=a_{\phi \phi}+\mathrm{W}^{-1}, A_{z}=a_{z z}+\mathrm{W}^{-1}, K_{I}=\mathrm{W}^{-1}+(k-\beta) I / 3, \quad I=a_{r r}+a_{\phi \phi}+a_{z z},
\end{aligned}
$$

$\operatorname{Re}=\left(\rho u_{H} l_{H}\right) / \eta_{0}-$ число Рейнольдса, $\mathrm{W}=\left(\tau_{0} u_{H}\right) / l_{H}-$ число Вайсенберга,

$\eta_{0}, \tau_{0}-$ начальные значения сдвиговой вязкости и времени релаксации.

Следуя монографии [9], работам [10-12], будем искать эти течения несжимаемой полимерной жидкости в “кармановском” виде, т.е. будем искать у системы (1)-(14) решения вида $[9,10]$ :

$$
\begin{aligned}
& u(t, r, \phi, z)=r F(t, z)+o(r), \\
& v(t, r, \phi, z)=r G(t, z)+o(r), \\
& w(t, r, \phi, z)=H(t, z)+O(r), \\
& P(t, r, \phi, z)=D(t, z)+\left(r^{2} / 2\right) K(t)+o\left(r^{2}\right), \\
& a_{r r}(t, r, \phi, z)=A_{0}(t, z)+\left(r^{2} / 2\right) A(t, z)+o\left(r^{2}\right), \\
& a_{\phi \phi}(t, r, \phi, z)=A_{0}(t, z)+\left(r^{2} / 2\right) \Phi(t, z)+o\left(r^{2}\right), \\
& a_{z z}(t, r, \phi, z)=C(t, z)+O(r), \\
& a_{r \phi}(t, r, \phi, z)=\left(r^{2} / 2\right) U(t, z)+o\left(r^{2}\right), \\
& a_{r z}(t, r, \phi, z)=r B(t, z)+o(r), \\
& a_{\phi z}(t, r, \phi, z)=r Q(t, z)+o(r), \\
& \tilde{L}(t, r, \phi, z)=r L(t, z)+o(r), \\
& \tilde{M}(t, r, \phi, z)=r M(t, z)+o(r), \\
& \tilde{N}(t, r, \phi, z)=N(t, z)+O(r) .
\end{aligned}
$$

Здесь $o(r)=r^{2}\{\ldots\}(t, z)+\ldots, O(r)=r\{\ldots\}(t, z)+\ldots, o\left(r^{2}\right)=r^{3}\{\ldots\}(t, z)+\ldots$. 
Заметим, что у компонент $a_{r r}$ и $a_{\phi \phi}$ главные члены разложения одинаковы в силу системы (1)-(14).

В реальных течениях мы имеем дело не с бесконечной пластиной $z=0$, a с конечным диском радиуса $r_{0}\left(l_{H}=r_{0}\right)$. Поэтому, как и для вязкой несжимаемой жидкости (см. [9] и описанную там дискуссию по этому поводу), разложения (15), по-видимому, справедливы при малых $r: 0<r \ll r_{*}=1$. В этой связи обозначения $o(r), O(r)$ и т.д. вполне приемлемы. Заметим также, что разложения (15) носят, конечно же, формальный характер до тех пор, пока не доказана сходимость соответствующих рядов.

Подставляя представление (15) в систему (1)-(14), собирая и затем зануляя коэффициенты при соответствующих степенях $r$, получим следующую нестационарную систему для определения агрегатов $F, G, H, D, A_{0}$, $A, \Phi, C, U, B, Q, L, M, N$ :

$$
\begin{aligned}
& H_{z}=-2 F \\
F_{t}+ & H F_{z}+F^{2}-G^{2}+K(t)=\frac{1}{\operatorname{Re}}\left(\frac{3 A-\Phi}{2}+B_{z}\right)+\sigma_{m}\left((1+N) L_{z}-2 M^{2}\right), \\
& G_{t}+H G_{z}+2 F G=\frac{1}{\operatorname{Re}}\left(2 U+Q_{z}\right)+\sigma_{m}(2 L M+(1+N) m), \\
& H_{t}+H H_{z}+D_{z}=\frac{1}{\operatorname{Re}}\left(2 B+C_{z}\right) \\
& \left(A_{0}\right)_{t}+H\left(A_{0}\right)_{z}-2\left(A_{0}+\mathrm{W}^{-1}\right) F+l A_{0}+\beta A_{0}^{2}=0 \\
& A_{t}+H A_{z}-4 B F_{z}+l A+2 \beta\left(A_{0} A+B^{2}\right)+\frac{k-\beta}{3}(A+\Phi) A_{0}=0 \\
& \Phi_{t}+H \Phi_{z}-4 Q G_{z}+l \Phi+2 \beta\left(A_{0} \Phi+Q^{2}\right)+\frac{k-\beta}{3}(A+\Phi) A_{0}=0 \\
& U_{t}+H U_{z}-2\left(B G_{z}+Q F_{z}\right)+l U+2 \beta\left(A_{0} U+B Q\right)=0 \\
& B_{t}+H B_{z}+2 F B-\left(\mathrm{W}^{-1}+C\right) F_{z}+l B+\beta\left(A_{0}+C\right) B=0 \\
& Q_{t}+H Q_{z}-\left(\mathrm{W}^{-1}+C\right) G_{z}+l Q+\beta\left(A_{0}+C\right) Q=0 \\
& C_{t}+H C_{z}+l C+\beta C^{2}=0, \\
& N_{z}=-2 L, \\
& L_{t}+H L_{z}-(1+N) F_{z}-b_{m} L_{z z}=0 \\
& M_{t}+H M_{z}-(1+N) G_{z}-b_{m} M_{z z}=0 \\
& N_{t}+H N_{z}-(1+N) H_{z}-b_{m} N_{z z}=0 \\
&
\end{aligned}
$$


где $l=\mathrm{W}^{-1}+(k-\beta)\left(2 A_{0}+C\right) / 3, \quad m=M_{z}$.

На самом деле после подстановки представлений (15) в систему (1)(14) мы должны были бы получить бесконечную систему соотношений, из которой далее мы выделили замкнутую подсистему уравнений (16)-(30).

В силу условий прилипания при $z=0$ имеем

$$
\begin{aligned}
& F(t, 0)=H(t, 0)=0, \\
& G(t, 0)=\Lambda(t) .
\end{aligned}
$$

Для компонент магнитного поля при $z=0$ получаем $[14,15]$

$$
\begin{aligned}
& L(t, 0)=M(t, 0)=0, \\
& N(t, 0)=\chi_{0}^{(1)}, \\
& N_{z}(t, 0)=N_{z z}(t, 0)=L_{z}(t, 0)=0, \\
& M_{z}(t, 0)=m_{0} .
\end{aligned}
$$

Здесь $\chi_{0}^{(1)}$ - магнитная восприимчивость при комнатной температуре в области $S_{1}$ (область $S_{1}$ тоже является магнетиком, см. рис.1); $m_{0}$ - некоторая постоянная.

Заметим также, что в силу (27) уравнение (28) есть следствие из уравнения (30), то есть уравнение (28) можно опустить.

Пусть свободная граница задается уравнением

$$
z=h(t, r) \text {. }
$$

Следуя [24], получим для свободной границы $z=h$ условие

$$
h_{t}+u h_{r}-w=0 \text {. }
$$

Как было оговорено выше, приближенное решение мы рассматриваем в цилиндрической зоне, представляющей из себя окрестность оси вращения диска малого радиуса. В связи с этим, разумным представляется предположить, что, как и в $[11,12]$, высота слоя жидкости является постоянной

$$
h=h_{*}=\text { const } .
$$

Следовательно, из (33) получаем

$$
H\left(t, h_{*}\right)=0 .
$$

Важно отметить, что постоянная $h_{*}$ является неизвестной величиной, а условие (38) служит для ее определения. Математически сделанное предположение означает, что мы ограничились первым слагаемым разложения функции $h(t, r)$ по радиальной компоненте. 
Будем полагать, что область $S_{2}$ занята средой с нулевой проводимостью $\sigma$, то есть $b_{m}=0$ в $S_{2}$. Тогда в области $S_{2}[13,20]$ имеем

$$
\begin{aligned}
& \Delta_{r, \phi, z} \tilde{L}^{(2)}-\frac{2}{r^{2}} \frac{\partial \tilde{M}^{(2)}}{\partial \phi}-\frac{\tilde{L}^{(2)}}{r^{2}}=0, \\
& \Delta_{r, \phi, z} \tilde{M}^{(2)}+\frac{2}{r^{2}} \frac{\partial \tilde{L}^{(2)}}{\partial \phi}-\frac{\tilde{M}^{(2)}}{r^{2}}=0, \\
& \Delta_{r, \phi, z} \tilde{N}^{(2)}=0
\end{aligned}
$$

где $\tilde{L}^{(2)}, \tilde{M}^{(2)}, 1+\tilde{N}^{(2)}$ - компоненты вектора напряженности магнитного поля в области $S_{2}$.

Полагая, что в области $S_{2}$ выполняются соотношения

$$
\begin{aligned}
& \tilde{L}^{(2)}(t, r, \phi, z)=r L^{(2)}(t, z)+o(r), \quad \tilde{M}^{(2)}(t, r, \phi, z)=r M^{(2)}(t, z)+o(r), \\
& \tilde{N}^{(2)}(t, r, \phi, z)=N^{(2)}(t, z)+O(r),
\end{aligned}
$$

получим из (39)-(41):

$$
\begin{aligned}
& L^{(2)}=\hat{L}^{(2)}(t), \\
& M^{(2)}=\hat{M}^{(2)}(t), \\
& N^{(2)}=\hat{N}^{(2)}(t) .
\end{aligned}
$$

При получении соотношений (42)-(44) мы полагали, что при $z \rightarrow \infty$ величины $L^{(2)}, M^{(2)}, N^{(2)}$ ограничены. Заметим, что в силу [14,15] мы имеем

$$
L\left(t, h_{*}\right)=\hat{L}^{(2)}(t), \quad M\left(t, h_{*}\right)=\hat{M}^{(2)}(t), \quad \hat{N}^{(2)}(t)=\frac{1+N\left(t, h_{*}\right)}{1+\chi_{0}^{(2)}}-1 .
$$

Здесь $\chi_{0}^{(2)}$ - магнитная восприимчивость в области $S_{2}$ при комнатной температуре (как и область $S_{1}$, область $S_{2}$ также является магнетиком, см. рис.1).

\section{2. Стационарные решения, описывающие течения "кармановского" ти- па вблизи оси $\boldsymbol{r}=0$}

Рассмотрим теперь стационарный случай, т.е. будем считать, что искомые функции не зависят от времени. Обозначая штрихами производные по переменной $z$, получим из (16)-(30) следующую систему обыкновенных дифференциальных уравнений (см. также (21)-(36)). Здесь мы ввели вспомогательную функцию $m$ для того, чтобы получить систему уравнений первого порядка. 
Вихревые стационарные структуры Кармана в магнитогидродинамических ...

$H^{\prime}=-2 F, \quad H(0)=0$,

$\left(H^{2}-\delta\right) F^{\prime}=H\left(G^{2}-F^{2}+\frac{3 A-\Phi}{2 \operatorname{Re}}+\sigma_{m}\left((N+1) L^{\prime}-2 M^{2}\right)-K_{0}\right)-$

$-B \frac{(2 F+\tilde{l})}{\operatorname{Re}}, \quad F(0)=0$,

$\left(H^{2}-\delta\right) B^{\prime}=\left(\mathrm{W}^{-1}+C\right)\left(G^{2}-F^{2}+\frac{3 A-\Phi}{2 \operatorname{Re}}+\sigma_{m}\left((N+1) L^{\prime}-2 M^{2}\right)-K_{0}\right)-$

$-H(2 F+\tilde{l}) B, \quad B(0)=b_{0}$,

$\left(H^{2}-\delta\right) G^{\prime}=2 H\left(\frac{U}{\operatorname{Re}}+\sigma_{m}\left(L M+\frac{N+1}{2} m\right)-F G\right)-Q \frac{\tilde{l}}{\operatorname{Re}}, \quad G(0)=\Lambda_{0}$,

$\left(H^{2}-\delta\right) Q^{\prime}=2\left(\mathrm{~W}^{-1}+C\right)\left(\frac{U}{\operatorname{Re}}+\sigma_{m}\left(L M+\frac{N+1}{2} m\right)-F G\right)-H Q \tilde{l}, \quad Q(0)=q_{0}$,

$\left(H\left(\mathrm{~W}^{-1}+A_{0}\right)\right)^{\prime}=-\hat{l} A_{0}, \quad A_{0}(0)=a_{00}$,

$\left(H\left(\mathrm{~W}^{-1}+C\right)\right)^{\prime}=-\hat{l}_{0} C-2\left(\mathrm{~W}^{-1}+C\right) F, \quad C(0)=c_{0}$,

$\left(\frac{H^{2}}{2}+D-\frac{C}{\operatorname{Re}}\right)=\frac{2 B}{\operatorname{Re}}, \quad D(0)=d_{0}$,

$H\left(H^{2}-\delta\right) A^{\prime}=4 B H\left(G^{2}-F^{2}+\frac{3 A-\Phi}{2 \operatorname{Re}}+\sigma_{m}\left((N+1) L^{\prime}-2 M^{2}\right)-K_{0}\right)-$

$-4 B^{2} \frac{2 F+\tilde{l}}{\operatorname{Re}}-\hat{l}_{1} A\left(H^{2}-\delta\right)-2 \beta B^{2}\left(H^{2}-\delta\right)-\frac{k-\beta}{3}(A+\Phi) A_{0}\left(H^{2}-\delta\right)$,

$A(0)=a_{0}$,

$H\left(H^{2}-\delta\right) \Phi^{\prime}=8 Q H\left(\frac{U}{\operatorname{Re}}+\sigma_{m}\left(L M+\frac{N+1}{2} m\right)-F G\right)-4 Q^{2} \frac{\tilde{l}}{\operatorname{Re}}-$

$-\hat{l}_{1} \Phi\left(H^{2}-\delta\right)-2 \beta Q^{2}\left(H^{2}-\delta\right)-\frac{k-\beta}{3}(A+\Phi) A_{0}\left(H^{2}-\delta\right), \Phi(0)=\varphi_{0}$,

$H\left(H^{2}-\delta\right) U^{\prime}=4 H B\left(\frac{U}{\operatorname{Re}}+\sigma_{m}\left(L M+\frac{N+1}{2} m\right)-F G\right)+$

$+2 Q H\left(G^{2}-F^{2}+\frac{3 A-\Phi}{2 \operatorname{Re}}+\sigma_{m}\left((N+1) L^{\prime}-2 M^{2}\right)-K_{0}\right)-$

$-4 Q B \frac{F+\tilde{l}}{\operatorname{Re}}-U\left(H^{2}-\delta\right) \hat{l}_{1}-2 \beta B Q\left(H^{2}-\delta\right), U(0)=u_{0}$, 


$$
\begin{aligned}
& N^{\prime}=-2 L, \quad N(0)=\chi_{0}^{(1)}, \\
& L^{\prime}=\frac{H}{b_{m}} L-\frac{F}{b_{m}}(N+1), \quad L(0)=0, \\
& M^{\prime}=m, M(0)=0, \\
& m^{\prime}=\frac{H}{b_{m}} m-\frac{(N+1) G^{\prime}}{b_{m}}, \quad m(0)=m_{0} .
\end{aligned}
$$

Здесь $\delta=\left(\mathrm{W}^{-1}+C\right) / \mathrm{Re}$,

$$
\begin{aligned}
& \tilde{l}=(2 k+\beta) A_{0} / 3+(k+2 \beta) C / 3+\mathrm{W}^{-1}, \\
& \hat{l}=(2 k+\beta) A_{0} / 3+(k-\beta) C / 3+\mathrm{W}^{-1}, \\
& \hat{l}_{0}=2(k-\beta) A_{0} / 3+(k+2 \beta) C / 3+\mathrm{W}^{-1}, \\
& \hat{l}_{1}=\mathrm{W}^{-1}+2(k+2 \beta) A_{0} / 3+(k-\beta) C / 3 .
\end{aligned}
$$

При этом $K(t)=$ const $=K_{0}, \Lambda(t)=$ const $=\Lambda_{0}$.

Основной трудностью полученной задачи является краевая точка $z=0$. В самом деле, краевое условие $H(0)=0$ приводит к вырождению уравнений (50), (51), (53)-(55), которые отсутствуют в аналогичной задаче для ньютоновской жидкости $[11,12]$. Отсюда следует, что, в отличие от уравнений «обычной» вязкой жидкости [11], задача не может иметь решений при произвольных краевых условиях. Рассматривая уравнения (50), (51), (53)-(55) при $z=0$, получим необходимые условия существования решения задачи:

$$
\begin{array}{ll}
a_{00}=0, & c_{0}=0,
\end{array}
$$

Уравнение (52) служит для определения $D(z)$ - давления на оси $r=0$ :

$$
D(z)=-\frac{H^{2}(z)}{2}+\frac{C(z)}{\operatorname{Re}}+\frac{2}{\operatorname{Re}} \int_{0}^{z} B(s) d s+d_{0} .
$$

Из уравнения (46) следует, что $\left.F^{\prime}(z)\right|_{z=0}=b_{0}$. Тогда из (45) $\left.H^{\prime \prime}(z)\right|_{z=0}=$ $=-2 b_{0}$. Учитывая условия $H(0)=0,\left.H^{\prime}(z)\right|_{z=0}=0$, получаем, что в окрестности нуля $H(z)$ имеет знак, обратный знаку $b_{0}$.

Легко заметить, что из (51) с учетом (45) и (61) следует $C(z) \equiv 0$. 


\section{3. Поиск численных стационарных решений}

В этом разделе для простоты будем полагать, что $k=\beta$. Из-за упомянутой ранее особенности задачи, ее численный расчет затруднителен, поскольку $z=0$ в силу условия $H(0)=0$ является сингулярной для уравнений (50), (51), (53)-(55). Чтобы избавиться от особенности в крайней точке, введем вспомогательную независимую переменную $\xi$ по правилу

$$
\frac{d z}{d \xi}=H\left(H^{2}-\delta\right)
$$

Предполагая, что для некоторой точки $\xi^{\prime}$ выполняется условие $z\left(\xi^{\prime}\right)=0$, положим

$$
\begin{aligned}
& \frac{d H}{d \xi}=\frac{d H}{d z} \frac{d z}{d \xi}=-2 F H\left(H^{2}-\delta\right), \\
& \frac{d F}{d \xi}=H\left(H^{2}-\delta\right) \frac{d F}{d z}, \ldots, \frac{d m}{d \xi}=H\left(H^{2}-\delta\right) \frac{d m}{d z}, \\
& \left.z\right|_{\xi=\xi^{\prime}}=0,\left.H\right|_{\xi=\xi^{\prime}}=0, \ldots,\left.m\right|_{\xi=\xi^{\prime}}=m_{0} .
\end{aligned}
$$

Задача (62)-(65) уже не имеет вырождающихся уравнений в начальной точке. Поэтому она в целом поддается численному расчету конечно-разностными схемами первого порядка. Однако очевидно, что точка $z=0, H=0$ является положением равновесия в фазовой плоскости $(z, H)$, т.к. производные искомых функций $z$ и $H$ равняются нулю при $\xi=\xi^{\prime}$. Поэтому для того, чтобы найти неизвестные величины задачи (45)-(59) как функции от $z$, необходимо в фазовом пространстве неизвестных функций найти траекторию, попадающую в краевые условия (65), т.е. бесконечно приближающуюся к точке, задающей эти условия. Поскольку при малых положительных $H$ производная $d z / d \xi$ отрицательна, функция $z(\xi)$ на такой траектории будет достигать значения $z\left(\xi^{\prime}\right)=0$ при $\xi^{\prime}=+\infty$.

Определим следующие значения параметров задачи, которые назовем базовыми:

$$
\begin{aligned}
& \operatorname{Re}=1, \quad \mathrm{~W}=1, \quad \beta=0.5, \quad b_{0}=-1, \quad q_{0}=1, \quad K_{0}=1, \\
& \Lambda_{0}=1, \quad \sigma_{m}=1, \quad b_{m}=1, \quad \chi_{0}^{(1)}=1, \quad m_{0}=1 .
\end{aligned}
$$

Введем для системы (62)-(65) разностную сетку $\xi_{j}=\tau j, j \in \mathbf{Z}, z_{j}=z\left(\xi_{j}\right)$ и т.д. Производные неизвестных функций будет аппроксимировать по правилу

$$
\frac{d z\left(\xi_{j}\right)}{d \xi}=\frac{z_{j+1}-z_{j}}{\tau}, \ldots
$$


Будем использовать постоянное значение $\tau=10^{-4}$. Здесь положительные $j$ соответствуют значениям $z$, лежащим левее (ближе к нулю) некоторой начальной точки $z_{0}=z\left(\xi_{0}\right)>z_{j}$, а отрицательные $j-$ значениям $z_{j}>z_{0}$, и при $\xi=-\infty$ траектория достигает свободной поверхности $z=h_{*}$, на которой $H\left(h_{*}\right)=0$.

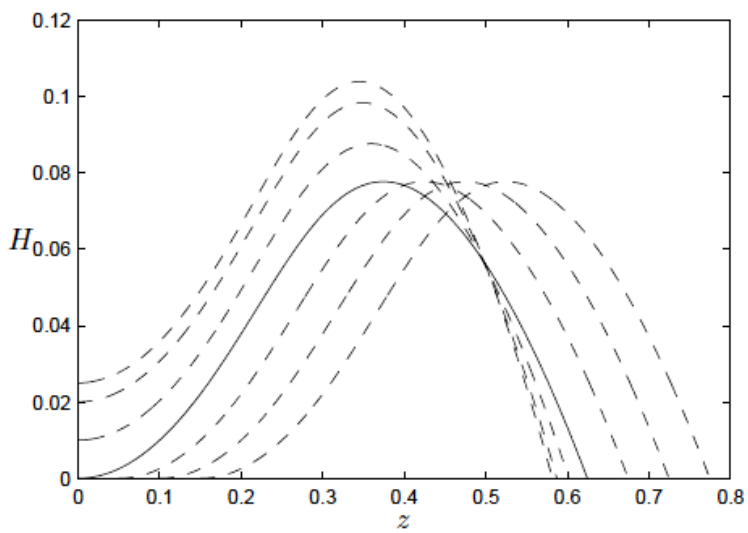

Рис.2. Траектории решений уравнений (62)-(65) на плоскости $(z, H)$.

Рассмотрим траекторию системы (62)-(65) в фазовом пространстве $\mathbf{X}=\left(z, H, F, B, G, Q, A_{0}, A, \Phi, U, N, L, M, m\right)$. Рис.2 показывает пример нескольких таких фазовых траекторий, спроектированных на плоскость $(z, H)$, проходящих рядом с началом координат этой плоскости. Из них наиболее близкой к искомому решению является траектория, обозначенная сплошной линией - она выпущена вправо по $z$ из точки $\mathbf{x}_{m}=\left(0,10^{-5}, 0, b_{0}, \Lambda_{0}, q_{0}, 0, a_{0}\right.$, $\left.\varphi_{0}, u_{0}, \chi_{0}^{(1)}, 0,0, m_{0}\right)$. В силу непрерывности правых частей уравнений, можно утверждать, что среди подобных траекторий будет стремящаяся к точке, соответствующей краевым условиям задачи (62)-(65). Для того чтобы еe найти, из некоторой стартовой точки $\mathbf{x}^{0}=\left(z^{0}, H^{0}, F^{0}, B^{0}, G^{0}, Q^{0}, A_{0}^{0}, A^{0}, \Phi^{0}\right.$, $\left.U^{0}, N^{0}, L^{0}, M^{0}, m^{0}\right)$ выпустим численную траекторию в сторону $\xi \rightarrow+\infty$ до тех пор, пока либо $z$, либо $H$ не приблизятся к нулю. Мы останавливали расчет при выполнении одного из условий: $z=z_{J}<10^{-7}$ либо $H=H_{J}<10^{-7}$. Соответственно, координаты точки фазового пространства, в которой мы остановили траекторию, обозначим индексом $J$. Введем функцию ошибки

$$
\begin{aligned}
& T\left(\mathbf{x}^{0}\right)=\left|z_{J}\right|+\left|H_{J}\right|+\left|F_{J}\right|+\left|B_{J}-b_{0}\right|+\left|G_{J}-\Lambda_{0}\right|+\left|Q_{J}-q_{0}\right|+\left|A_{0 J}\right|+ \\
& +\left|A_{J}-a_{0}\right|+\left|\Phi_{J}-\varphi_{0}\right|+\left|U_{J}-u_{0}\right|+\left|N_{J}-\chi_{0}^{(1)}\right|+\left|L_{J}\right|+\left|M_{J}\right|+\left|m_{J}-m_{0}\right|,
\end{aligned}
$$


характеризующую отклонение полученной траектории от требуемых начальных данных. Поиск искомой траектории будем вести минимизацией функции $T$. Правда, пока мы корректно определили значения функции $T$ только для таких значений аргумента $\mathbf{x}^{0}$, у которого первые две координаты положительны $\left(z^{0}>0, H^{0}>0\right)$. Для того чтобы итерации численного метода поиска минимума корректно работали, необходимо доопределить функцию $T$ в областях $z^{0}<0$ и $H^{0}<0$ так, чтобы минимум такой функции совпадал с минимумом исходной. Для этого воспользуемся введенной ранее траекторией, выпущенной из точки $\mathbf{x}_{m}$. Поскольку эта траектория довольно близка к искомому решению, используем ее масштаб для доопределения $T$. Обозначим за $z_{m}$ значение $z>0$, при котором траектория, выпущенная вправо по $z$ из точки $\mathbf{x}_{m}$, пересекает гиперплоскость $H=0$. За $H_{m}$ примем максимальное значение $H$ на траектории, выходящей из $\mathbf{x}_{m}$. Так, для траектории, обозначенной сплошной линией на рис.2, имеем $z_{m}=0.625$ и $H_{m}=0.078$. Тогда доопределим

$$
T\left(x^{0}\right)=\left\{\begin{array}{l}
z_{m}-z^{0}, \quad z^{0}<0, H^{0} \geq 0, \\
H_{m}-H^{0}, H^{0}<0 .
\end{array}\right.
$$

Минимум функции $T$ мы будем искать симплекс-методом НелдераМида при помощи функции fminsearch пакета программ MATLAB. За начальную точку метода возьмем точку $\mathbf{x}_{m}$. Точку $\mathbf{x}^{0}$, отвечающей минимуму функции $T$, будем считать точкой, лежащей на искомой траектории - решении (62)-(65). Выпустим из этой точки траекторию в обе стороны $(\xi \rightarrow \pm \infty)$ до тех пор, пока с обеих сторон траектория не приблизится к значениям $H=0$. Левая точка будет соответствовать значению $z=0$, а правая - значению $h_{*}=z\left(\xi_{*}\right)$.

На рис.3 приведены графики решений задачи (62)-(65) для базовых значений параметров, найденных описанным выше методом. Здесь мы показываем значения для компонент скорости $H, F, G$ и компонент напряженности магнитного поля $L, M, N$ согласно разложению (15). Хотелось бы отметить характерный профиль компоненты $F$, которая с ростом $z$ меняет знак с отрицательного на положительный. Другими словами, с ростом высоты режим течения меняется с циклонического (поток к центру вращения) на антициклонический (поток в направлении от центра вращения). Также поведение скорости потока является прямым следствием уравнения (45) и краевых условий (31), (38). 

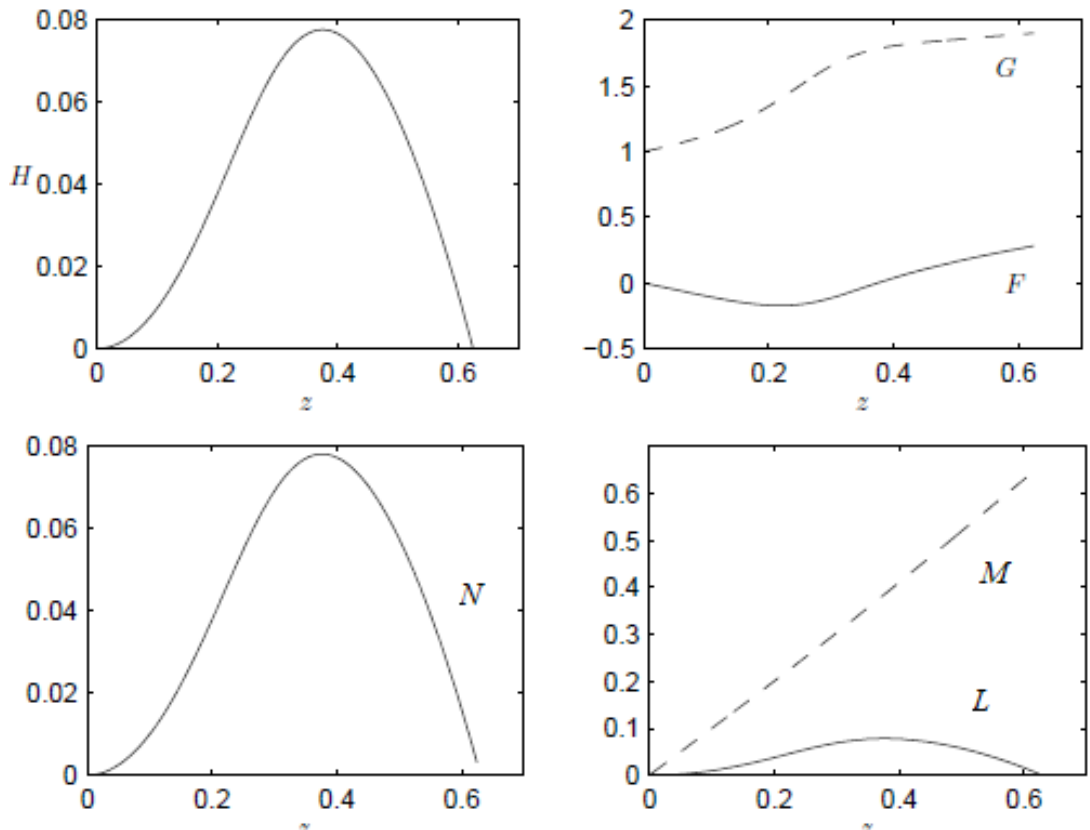

Рис.3. Решение для базовых значений параметров.
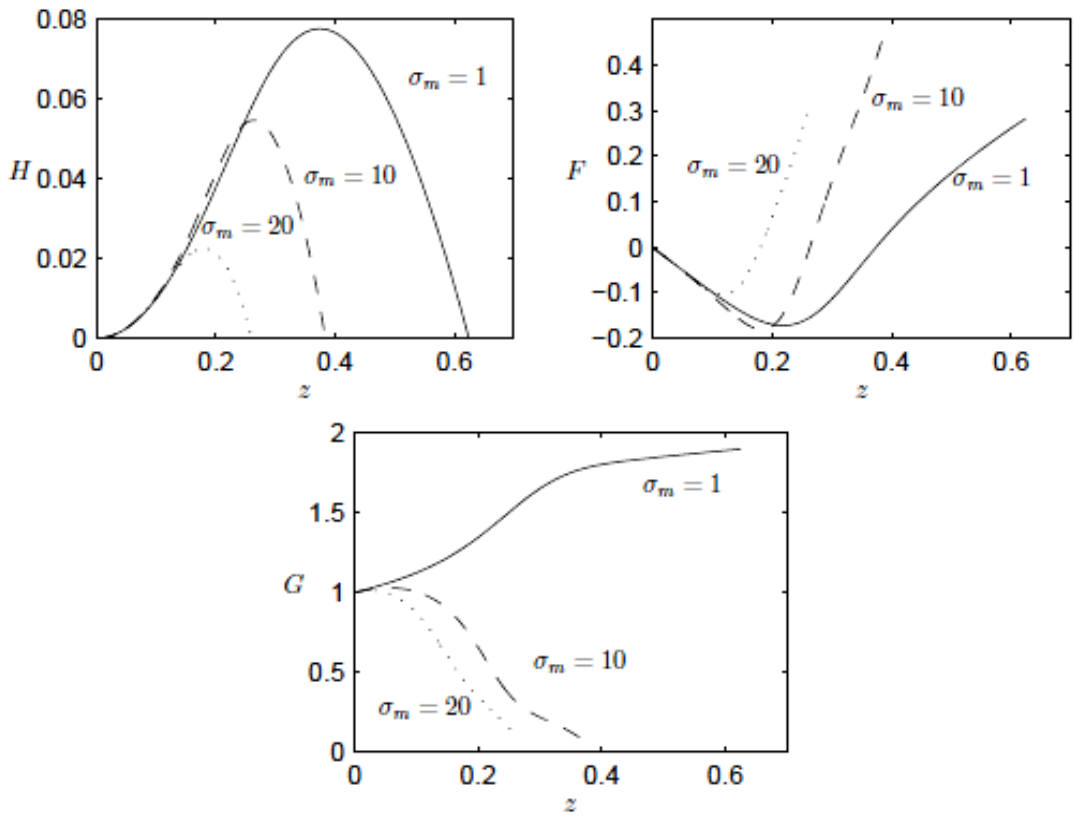

Рис.4. Скорость жидкости при различных значениях $\sigma_{m}$.

Рассмотрим теперь несколько примеров решений при других значениях параметров. На рис.4 изображены графики компонент скорости $H, F$ и $G$ при различных значениях коэффициента магнитного давления $\sigma_{m}$. Видно, что 

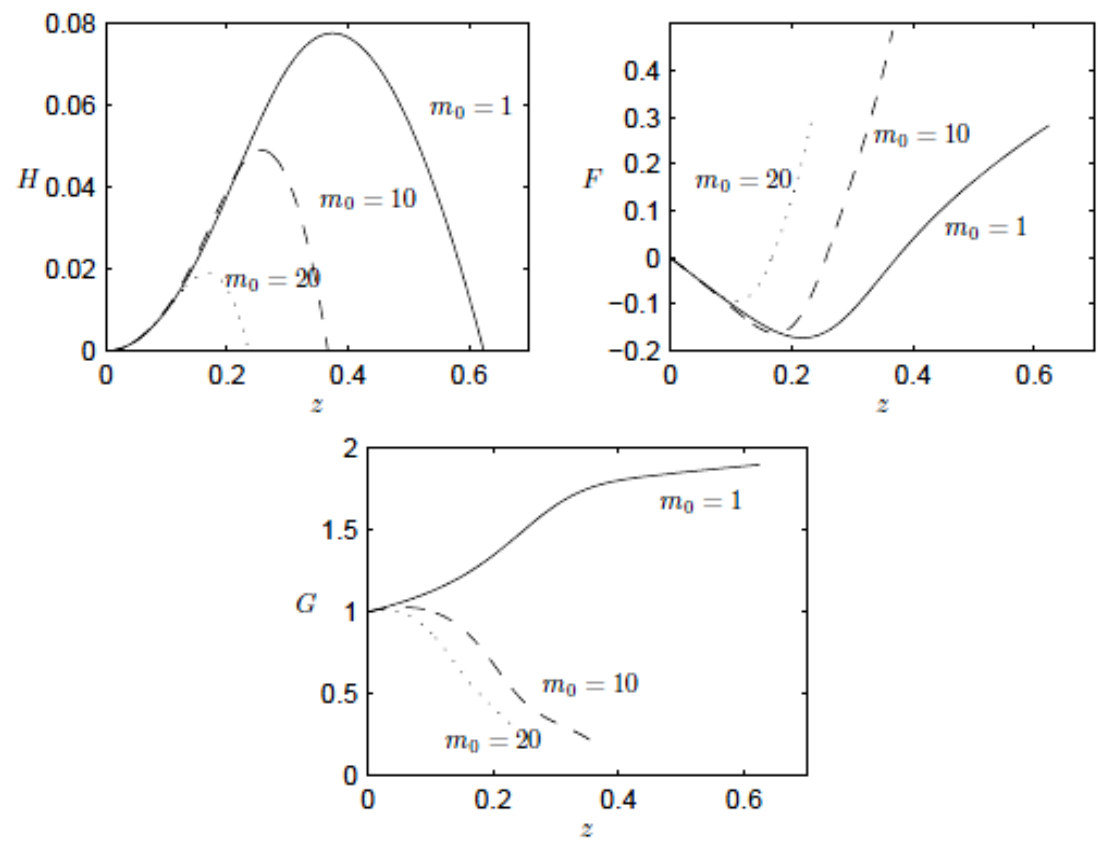

Рис.5. Скорость жидкости при различных значениях $m_{0}$.

увеличение параметра $\sigma_{m}$ ведет к уменьшению высоты $h *$ и одновременно к уменьшению величины вертикальной скорости жидкости. Более существенный эффект изменение параметра оказывает на угловую компоненту скорости $G$. В зависимости от значения $\sigma_{m}$ угловая скорость может как расти с увеличением высоты, так и уменьшаться. Сходное влияние имеет и значение начального данного $m_{0}$ на рис.5. Влияние магнитной восприимчивости $\chi_{0}^{(1)}$ на профили скорости, в свою очередь, является более сложным (см. рис.6). Так, графики функции $H$ показывают, что при изменении параметра средний рост вертикальной скорости необязательно ведет к увеличению высоты зоны вращательного движения. Например, при $\chi_{0}^{(1)}=10$ глубина жидкости меньше, чем при $\chi_{0}^{(1)}=1$ и больше, чем при $\chi_{0}^{(1)}=20$, однако же значения вертикальной скорости жидкости при $\chi_{0}^{(1)}=10$ больше, чем для двух других значений. Также на этих графиках видно, что при некоторых значениях параметров поведение угловой скорости может стать немонотонным по высоте. Наконец, на рис.7 показаны решения при различных значениях магнитного числа Рейнольдса $\operatorname{Re}_{m}$ (или параметра $b_{m}=1 / \operatorname{Re}_{m}$ ). Из основных особенностей здесь хотелось бы отметить график для $H$, имеющий несколько максимумов (и, соответственно, несколько переходов от циклонического режима к антициклоническому на графиках для $F$ ), а также смену знака функции $G$ по высоте. Т.е. за счет работы магнитного поля можно 
остановить вращение жидкости и даже закрутить ее в сторону, противоположную направлению вращения диска.
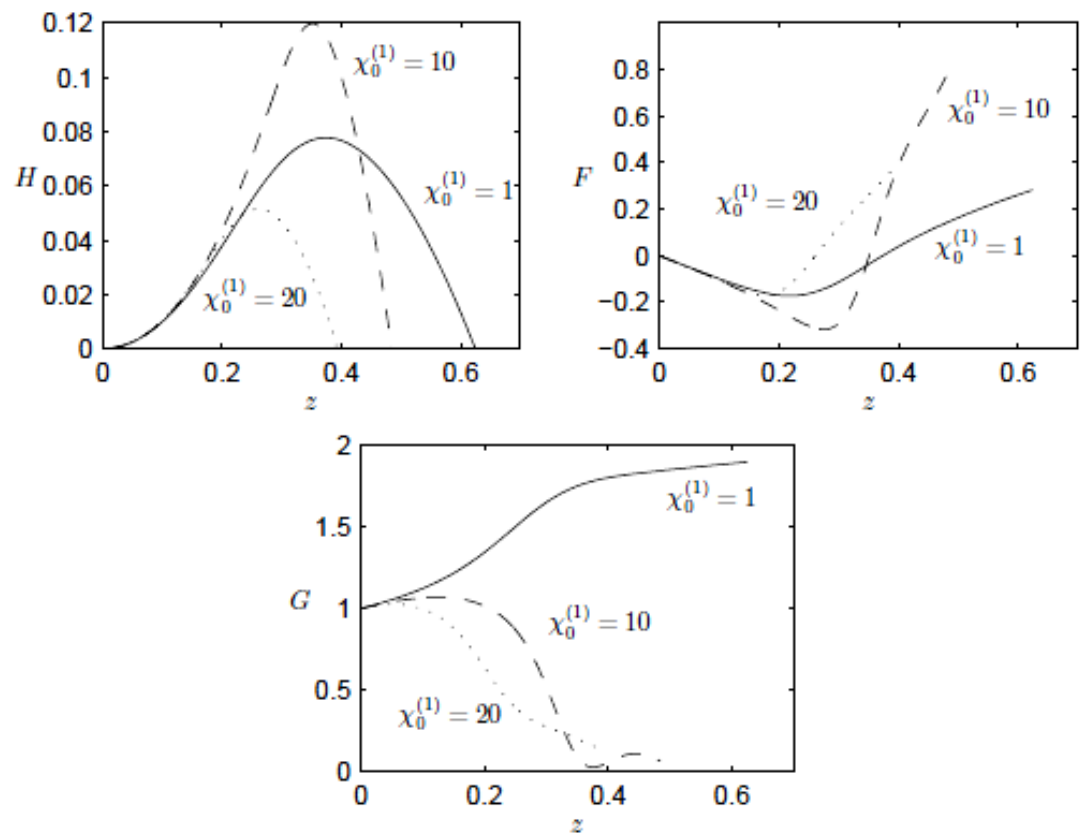

Рис.6. Скорость жидкости при различных значениях $\chi_{0}^{(1)}$.
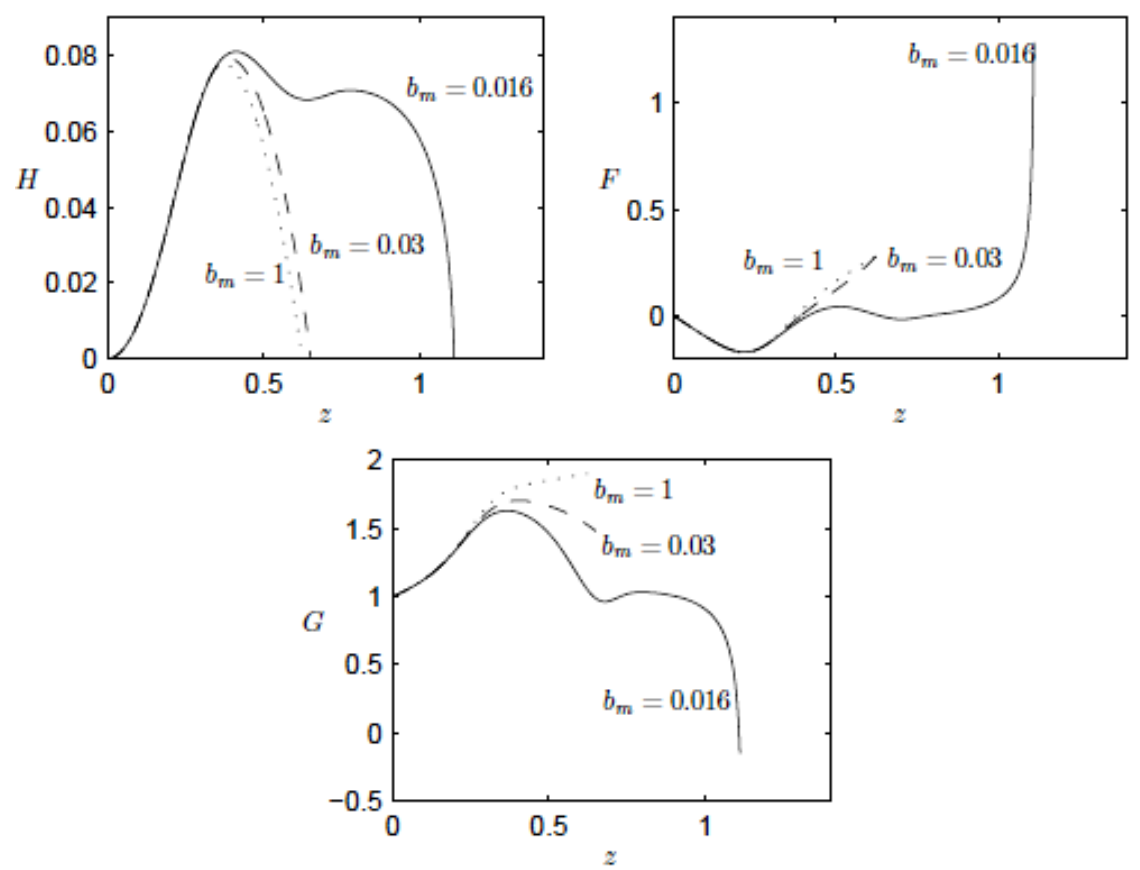

Рис.7. Скорость жидкости при различных значениях $b_{m}$. 


\section{Заключение}

В работе рассмотрен вариант реологической модели Виноградова-Покровского с учетом воздействия магнитного поля. Сформулирована задача о движении вязкоупругой жидкости конечной толщины над горизонтальным вращающимся диском. Для исследования стационарных режимов течения использовано представление для приближенного решения, сходное с автомодельным решением Кармана для вязкой жидкости. Наконец, нами предложен численный метод поиска стационарных решений задачи, позволяющий найти решения в окрестности сингулярной точки - поверхности диска.

При помощи предложенного нами метода мы рассмотрели стационарные решения задачи при различных значениях параметров. Так, было отмечено, что поток жидкости на разной высоте имеет разное направление по радиальной компоненте. Подобное поведение является следствием постановки задачи, которая предполагает стационарное течение с постоянной высотой слоя жидкости. Такая смена режима течения от циклонического (в направлении от оси вращения) к антициклоническому (к оси вращения) может происходить от одного до нескольких раз, в зависимости от значений параметров. Еще из интересных особенностей решений стоит отметить, что изменение части параметров одновременно приводят к возрастанию вертикальной скорости потока и к понижению верхней границы. Наконец, было показано, что работа магнитного поля может влиять на угловую скорость жидкости и даже менять направление вращения на противоположное вращению диска. Все вышеперечисленное показывает, что магнитное поле позволяет контролировать поток жидкости, существенно меняя его характеристики.

\section{СПИСОК ЛИТЕРАТУРЫ}

1. R. Ellahi. The effects of MHD and temperature dependent viscosity on the flow of nonNewtonian nanofluid in a pipe: Analytical solutions // Appl. Math. Model., 2013, v.37, №3, p.1451-1467.

2. Z. Khan, M.A. Khan, N. Siddiqui et. al. Solution of magnetohydrodynamic flow and heat transfer of radiative viscoelastic fluid with temperature dependent viscosity in wire coating analysis // PLOS One, 2018, v.13, №3, p.1-16.

3. T. Gul, S. Islam, R.A. Shah et al. Unsteady MHD thin film flow of an Oldroyd-B fluid over an oscillating inclined belt // PLOS One, 2015, v.10, №7, p.1-18.

4. L. Lie, L. Zhang. Axial MHD flow of generalized Oldroyd-B fluid due to two oscillating cylinders // Adv. mat. res., 2012, v.354, №1, p.83-86.

5. I. Khan, K. Fakhar, M.I. Anwar. Hydro-magnetic Rotating Flows of an Oldroyd-B Fluid in a Porous Medium // Sp. Top. and Rev. in Por. Med., 2012, v.3, №1, p.89-95.

6. А.М. Блохин, Р.Е. Семенко. Стационарные магнитогидродинамические течения не- 
сжимаемой полимерной жидкости в плоском канале // Вестник ЮУрГУ ММП, 2018, т.11, №4, c.41-54;

англ. пер.: A.M. Blokhin, R.E. Semenko. Stationary magnetohydrodynamical flows of nonisothermal polymeric liquid in the flat channel // Bullet. of the South Ural State Univ., Ser: Math. Model., Program. and Comput. Softw., v.11, №4, p.41-54.

7. Ю.А. Алтухов, А.С. Гусев, Г.В. Пьишнограй. Введение в мезоскопическую теорию текучих полимерных систем. - Барнаул: АлтГПА, 2012, 122 с.;

Iu.A. Altukhov, A.S. Gusev, G.V. Pyshnograi. Vvedenie v mezoskopicheskuiu teoriiu tekuchikh polimernykh sistem. - Barnaul: AltGPA, 2012, $122 \mathrm{~s}$.

8. T. von Karman. Über laminare und turbulente Reibung // ZAMM, 1921, v.1, №4, p.233-252.

9. Х. Гринспен. Теория вращающихся жидкостей. - Л.: Гидрометеоизд., 1975, 304 с.; H. Greenspan. The theory of rotating fluids. - Cambridge: Cambr. Univ. Press, 1968, 327p.

10. K. Stewartson. On the flow between two rotating coaxial discs // Proc. Cambridge Phil. Soc., 1953, v.49, №2, p.333-341.

11. С.В. Кострыкин, А.А. Хапаев, И.Г. Якушкин. Вихревые структуры в квазидвумерных течениях вязкой вращающейся жидкости // ЖЭТФ, 2011, т.139, №2, с.395-407;

англ. пер.: S.V. Kostrykin, A.A. Khapaev, I.G. Yakushkin. Vortex patterns in quasi-two-dimensional flows of a viscous rotating fluid // J. Exp. Theor. Phys., 2011, v.112, №2, p.344-354.

12. С.В. Кострыкин. Режимы стационарных течений в задаче об интенсивной вихревой циркуляции в тонком слое вязкой вращающейся жидкости // ЖЭТФ, 2018, т.154, №1, c.193-205;

англ. пер.: S.V. Kostrykin. Steady flow regimes in the problem of intense wind-driven circulation in a thin layer of viscous rotating fluid // J. Exp. Theor. Phys., 2018, v.127, №1, p.167-177.

13. А.Б. Ватажин, Г.А. Любимов, С.А. Регигер. Магнитогидродинамические течения в каналах. - М.: Наука, 1970, 674 с.;

A.B. Vatazhin, G.A. Liubimov, S.A. Regiger. Magnitogidrodinamicheskie techeniia v kanalakh. - M.: Nauka, 1970, 674 s.

14. К. Нордлинг, Д. Остерман. Справочник по физике для ученого и инженера. - СПб.: БХВ-Петербург, 2011, 528 с.;

C. Nordling, J. Osterman. Physics handbook for science and engineering. - Prof. Publ. House, 2006, 503 p.

15. С.Г. Калашников. Электричество. - М.: Физматлит, 2003, 624 с.;

S.G. Kalashnikov. Elektrichestvo. - M.: Fizmatlit, 2003. 624 s.

16. R. Bird, R. Armstrong, O. Hassager. Dynamics of polymeric liquids. - York: Wiley, 1987, 649p.

17. М. Дой, С. Эдвардс. Динамическая теория полимеров. - М.: Мир, 1998, 440 с.; M. Doi, S. Edwards. The theory of polymer dynamics. - Oxford: Clarendon press, 1986, 391p.

18. А.М. Блохин, Н.В. Бамбаева. Стационарные решения уравнений несжимаемой вязкоупругой полимерной жидкости // ЖВМиМФ., 2014, т.54, №5, с.55-69;

англ. пер.: N.V. Bambaeva, A.M. Blokhin. Stationary solutions of equations of incompressible viscoelastic polymer liquid // Comp. Math. and Math. Phys., 2014, v.54, №5, p.874-899.

19. А.М. Блохин, А.С. Рудометова. Стационарные течения слабопроводящей несжимаемой полимерной жидкости между соосными цилиндрами // Сиб. журн. индустр. матем, 2017, т.20, №4, с.13-21; 
англ. пер.: A.M. Blokhin, A.S. Rudometova. Stationary flows of a weakly conducting incompressible polymeric liquid between coaxial cylinders // J. of Appl. and Industr. Math., 2017, v.11, №4, p.486-493.

20. А.М. Блохин, Р.Е. Семенко. Вихревое движение несжимаемой полимерной жидкости в цилиндрической приосевой зоне // Мех. жидк. и газа, 2018, №2, с.3-15;

англ. пер.: A.M. Blokhin, R.E. Semenko. Vortex motion of an incompressible polymer liquid in the cylindrical near-axial zone // Fluid dyn., 2018, v.53, №2, p.177-188.

21. Л.И. Седов. Механика сплошной среды. Т.1. - М.: Наука, 1970, 492 с.; англ. пер.: L.I. Sedov. A course in continuum mechanics, V.1. - Groningen: Wolters Noordhoff Publ., 1972, 309 p.

22. Л.Г. Лойцянский. Механика жидкости и газа. - М.: Наука, 1978, 677 с.;

L.G. Loitsianskii. Mekhanika zhidkosti i gaza. - M.: Nauka, 1978, 677 s.

23. Ши-и Бай. Введение в теорию течения сжимаемой жидкости. - М.: Изд-во иностр. литер., 1962, 412 с.;

Shih-i Pai. Introduction to the theory of compressible flow. - Princeton: D. Van Nostrand Co., 1959, $385 \mathrm{p}$.

24. А.М. Блохин, Р.Е. Семенко. Стационарные электрогидродинамические течения несжимаемых полимерных сред с сильным разрывом // Сиб. журн. чист. и прикл. мат., 2017, т.17, №2. с.3-12;

англ. пер.: A.M. Blokhin, R.E. Semenko. Stationary electrohydrodynamic flows of incompressible polymeric media with strong discontinuity // J. of Math. Sci., 2018, v.231, №2, p.143-152.

Поступила в редакцию 11.11.2019

После доработки 13.01.2020

Принята к публикации 27.01.2020 